\title{
Visuotactile apparent motion
}

\author{
Vanessa Harrar, Rebecca Winter, ANd Laurence R. HARris \\ Centre for Vision Research, York University, Toronto, Ontario, Canada
}

\begin{abstract}
This article compares the properties of apparent motion between a light and a touch with apparent motion between either two lights or two touches. Visual and tactile stimulators were attached to the tips of the two index fingers that were held apart at different distances. Subjects rated the quality of apparent motion between each stimulus combination for a range of stimulus onset asynchronies (SOAs). Subjects reported perceiving apparent motion between all three stimulus combinations. For light-light visual apparent motion, the preferred SOA and the direction threshold SOAs increased as the distance between the stimuli increased (consistent with Korte's third law of apparent motion). Touch-touch apparent motion also obeyed Korte's third law, but over a smaller range of distances, showing that proprioceptive information concerning the position of the fingers is integrated into the tactile motion system. The threshold and preferred SOAs for visuotactile apparent motion did not vary with distance, suggesting a different mechanism for multimodal apparent motion.
\end{abstract}

Apparent motion is the illusory perception of motion created by the discrete stimulation of points appropriately separated in space and time. Apparent motion can be experienced between pairs of lights, touches, or sounds (see Kolers, 1972, for a review). In 1915, Korte published a set of seminal studies showing that the optimal timing for experiencing visual apparent motion varies with the temporal and spatial separations of the stimuli and their duration and intensity. He expressed this as a series of equations. One such equation states that the stimulus onset asynchrony (SOA) between two lights that produces optimal apparent motion is proportional to the distance between them. Although Korte did not explicitly state this as a law, but rather as a relationship, the variation of SOA with distance has become known as his third law after Koffka (1931).

Korte's third law was based on observations of visual apparent motion, and it also holds for tactile apparent motion (Burtt, 1917b; Kirman, 1974a; Sherrick, 1968). Auditory apparent motion, on the other hand, does not follow Korte's third law (Burtt, 1917a, dark soundproof room with two speakers at $200 \mathrm{~cm}$ from the observer, $5^{\circ}-20^{\circ}$ apart, and SOAs from 6 to $54 \mathrm{msec}$; Strybel, Manligas, Chan, \& Perrott, 1990, dark audiometric, semianechoic, chamber with two speakers at $86 \mathrm{~cm}$ from the observer, $6^{\circ}-160^{\circ}$ apart, and SOAs from 0 to $500 \mathrm{msec}$ ). Since both visual and tactile apparent motion follow the same rule, they may share a single motion mechanism. If there were a common visual and tactile motion system, then stimulating vision at one location and touch at another might activate this common motion mechanism and produce a sensation of multimodal visuotactile apparent motion that should also follow Korte's third law.

Experimental evidence for multimodal apparent motion was first reported by Zapparoli and Reatto in 1969, who investigated apparent motion between lights and sounds. They used lights that were $12 \mathrm{~mm}$ in diameter, separated by $7.7 \mathrm{~cm}$, and that corresponded to an angular separation of $3^{\circ}$, with sounds either played through loudspeakers beside each light or delivered through headphones. When all four stimuli were played (a light and sound on the left played together, followed by a light and sound on the right played together), subjects reported a single object or an event with two attributes (audio and visual) moving. The moving object/event was described in various ways - for example, a "sonorous light" or a "luminous sound." When only two stimuli were used (e.g., a single light followed by a single sound), some subjects described perceiving intermodal apparent motion. One subject described "a light tunnel which grows longer and shorter while the sound passes through it" (Zapparoli \& Reatto, 1969, p. 262). Other subjects described their perception as a "causal nexus between the two stimuli" (the light appeared to cause the sound or vice versa). This causal description suggests that multimodal apparent motion appears to be more cognitive than unimodal apparent motion.

Because of the cognitive component, Allen and Kolers (1981) concluded that the phenomenon of intermodal apparent motion was not "true" apparent motion. They repeated Zapparoli and Reatto's (1969) experiments, placing LEDs at $1.5 \mathrm{~m}$ from the observer with a distance between them from $3^{\circ}$ to $11.5^{\circ}$ on a sagittal plane, and used headphones (tones only, presented monaurally) to facilitate and attempt to replicate Zapparoli and Reatto's multimodal apparent motion. Allen and Kolers's interstimulus intervals (ISIs) varied from 50 to $150 \mathrm{msec}$. They concluded that subjects' interpretations were highly idiosyncratic and that they required the subject to be willing and eager to perceive the apparent motion, whereas visual and auditory appar-

V. Harrar, vharrar@yorku.ca 
ent motion perception was automatic. We suggest that it is partially language limitations that caused the interpretation of a bimodal moving stimulus to appear "unconventional," and not necessarily a difference in the perception itself.

Allen and Kolers (1981) went on to suggest in their discussion that audiovisual may not be the best combination for studying multimodal apparent motion, but they did not go on to investigate other sensory combinations. Indeed, auditory apparent motion may be a special case, since it does not follow Korte's third law (Burtt, 1917a), and the calculations involved in assessing the spatial position of a sound are very different from those used by the visual and tactile systems, in which spatially separate stimuli are also separated on the receptor organ.

Visuotactile multimodal motion appears more feasible than audiovisual apparent motion since there are bimodal visuotactile neurons in ventral intraparietal cortex that respond to apparent motion in either modality and that have spatially aligned visual and tactile receptive fields (Duhamel, Colby, \& Goldberg, 1998). This provides a neural substrate for visuotactile motion processing. We therefore set out to investigate visuotactile apparent motion.

We first asked subjects to rate the quality of apparent motion between two lights, two touches, and between a light and a touch (Experiment 1). Our hypotheses were that the perception of apparent motion between the different stimulus combinations (light-light, touch-touch, light-touch) would vary with SOA and that the SOAs that would produce the more convincing percept of apparent motion would depend on the stimulus combination.

In Experiment 2, we looked at the effect of distance between the stimuli on the preferred SOA for visual, tactile, and visuotactile apparent motion. Does Korte's third law hold for all three stimulus combinations?

In Experiment 3, we looked at the effect of distance between the stimuli on the threshold SOA for discriminating the direction of visual, tactile, and visuotactile apparent motion. Although Korte (1915) showed that the SOA for evoking optimal apparent motion varied with distance, Lakatos and Shepard (1997) tested the effect of distance on threshold SOA for detecting the direction of apparent motion. They used a circular arrangement of 12 stimuli in which neighboring pairs - or pairs separated by one, two, or three stimuli - could be used to effectively increase the distance between the stimuli. They presented visual stimuli in the frontal plane in a $10-\mathrm{cm}$ diameter circle viewed at $75 \mathrm{~cm}$ with a visual angle of $8^{\circ}$. Tactile stimuli were presented in a circle on the palm of the hand or around the head-5.6-cm and $23-\mathrm{cm}$ diameter circles, respectively. Auditory stimuli were presented in a dark, audiometric test chamber through speakers evenly positioned in a circle around the head (91-cm diameter). They measured the minimum SOA between the stimuli at which the direction of motion could be detected and showed that the threshold SOA for detecting the direction of apparent motion also varied with distance for visual, tactile, and auditory apparent motion. Since auditory apparent motion does not follow Korte's law at preferred speeds (Burtt, 1917b; Strybel et al., 1990) but does at threshold speeds (Lakatos \& Shepard, 1997), separate mechanisms for preferred and threshold apparent motion are suggested. Therefore, our hypothesis for Experiment 3 was that, regardless of the results of Experiment 2, an increased distance between the stimuli would produce a corresponding increase in the threshold SOA for detecting the direction of visual, tactile, and visuotactile apparent motion.

\section{GENERAL METHOD}

\section{Subjects}

Subjects were volunteers from the graduate and undergraduate pool at York University. Some subjects were paid for their participation. All experiments were approved by the York Ethics Board. Experiment 1 had 6 subjects ( 1 female, all right-handed, mean age 31 ); Experiment 2 had 6 subjects ( 5 female, all but 1 female righthanded, mean age 20), and Experiment 3 had 7 subjects ( 3 female, all right-handed, mean age 27).

\section{Touch Stimulators}

Touch stimulators were made from small solenoids with attached probes, and were mounted in $4 \times 2 \times 2 \mathrm{~cm}$ wooden cups. When the solenoids were powered, the probe was pushed out. The probe extended about $1 \mathrm{~mm}$ from the edge of the cup and hit the skin surface with the force of a gentle tap spread over a surface area of about $1 \mathrm{~mm}^{2}$. Solenoids were controlled by appropriately amplified 5-volt signals from a CED1401 interface box (Cambridge Electronic Design, U.K.) that was controlled by a PC. The solenoids took $5 \mathrm{msec}$ to extend and to retract back into their wooden cups, as measured by a photocell positioned directly above the solenoid (experiments were arranged so that this delay did not affect any of the results). The wooden cups with touch stimulators inside were taped on the tip of each subject's index fingers and adjusted until the taps were of comparable intensity. Subjects also wore hearing protectors (David Clark Co., Model 10a) to block out the remaining slight click that was generated by the solenoids when activated. Stimulus duration was $40 \mathrm{msec}$.

\section{Visual Stimulation}

Visual stimulation was provided by green LEDs mounted on top of the wooden cups that contained the solenoids. They were controlled directly by 5-volt signals from a CED1401 interface box controlled by a PC. Stimulus duration was also $40 \mathrm{msec}$.

\section{Response Pedals}

Responses were made by means of two foot pedals monitored by the CED1401. The pedals were normally held down. Subjects responded by lifting either the left or the right foot.

\section{EXPERIMENT 1 Quality Judgments}

Allen and Kolers (1981) have thrown doubt on the very existence of multimodal apparent motion, but they only tested apparent motion between a light and a sound. In order to see whether subjects perceived apparent motion between lights and touches, we had subjects rate the quality of unimodal and multimodal apparent motion over a range of SOAs.

\section{Method}

Participants sat in a well-lit room, with their hands on the table in front of them, palms up. They maintained fixation on a point between their hands. Stimulators were fixed on each of their index fingers, which were held $5 \mathrm{~cm}$ apart at a distance of $50 \mathrm{~cm}$ from the eyes, for a visual angle of $5.7^{\circ}$. The three stimulus combinations (light-light, touch-touch, and light-touch) were randomly interleaved in a single session. Each stimulus pair was presented to the subject with a par- 
ticular SOA that was chosen at random from a predetermined set $(50$, $75,100,125,150,175,200,225,250,275,300 \mathrm{msec}$ ). This set was specifically chosen to include those values used by previous researchers (e.g., Allen \& Kolers, 1981; Burtt, 1917a, 1917b; Zapparoli \& Reatto, 1969). The stimuli alternated (with a given SOA), potentially creating apparent motion that bounced back and forth. After the apparent motion stimulus terminated, subjects gave an unspeeded response before receiving the next stimulus trial. Participants were instructed to indicate - by means of pressing the appropriate key on a key pad $(0-6)$ - the quality of the perceived motion, where 1 was poor, 3 was ok but not ideal, and 5 was excellent. Zero was used if the subject did not perceive any motion or if the instruction made no sense. Finally, 6 was available if the subject missed the stimulus (blinked, lost attention, fell asleep, etc.); however, a response of 6 was never used. Each of the 11 SOAs was repeated three times for each of the three stimulus pairs, for a total of 99 randomly presented stimulus pairs. The experiment took approximately $20 \mathrm{~min}$ to complete.

\section{Results}

The average quality rating for each subject, each stimulus type, and each SOA was determined. A repeated measures ANOVA (with Greenhouse-Geisser correction) was done, comparing the variation of quality judgments of apparent motion for light-light, touch-touch, and lighttouch across 11 SOAs. A significant main effect of stimulus type was found $[F(2,10)=6.21, p=.049]$, indicating that visual $(M=3.48, S E=.27)$, tactile $(M=3.12, S E=$ $.33)$, and visuotactile $(M=2.16, S E=.22)$ apparent motion were different (see Figure 1A). A significant main effect of SOA was also found $[F(10,50)=7.82, p=.009]$, indicating that the quality varied with SOA. Most interestingly, there was a significant interaction effect between stimulus type and SOA $[F(20,100)=3.60, p=.034]$, which showed that quality ratings for the three types of apparent motion varied differently with SOA.

In a post hoc ANOVA, we noted that at SOAs from 200 to $300 \mathrm{msec}$, the quality judgments of visual, tactile, and multimodal apparent motion did not differ from each other $[F(2,10)=1.29, p=.31]$. This range is where quality ratings were the highest for multimodal apparent motion. One can infer that the best multimodal apparent motion is comparable to visual apparent motion at a $200-300 \mathrm{msec}$ delay.

There were individual differences in the way subjects rated the quality of multimodal apparent motion (see Figure 1B). Most subjects never used the 0 response; that is, most subjects perceived at least some visuotactile apparent motion at all delays. One subject did frequently respond with a 0 (in Figure $1 \mathrm{~B}$, the line that goes below 1$)$, but only at low SOAs. Even this subject had an average response of 3 at higher SOAs. Overall, multimodal apparent motion and the tactile apparent motion were given worse quality ratings than visual apparent motion (maximum quality of multimodal 2.9, tactile 3.6, and visual 4.4).

\section{Discussion}

The results confirm that visuotactile multimodal apparent motion exists and is comparable to visual and tactile apparent motion at longer delays. Most subjects perceived some degree of multimodal apparent motion even at very short SOAs, and all perceived a reasonable degree of apparent motion at longer delays.

These results differ from those of Allen and Kolers (1981). None of their subjects perceived true multimodal apparent motion, although they tested audiovisual stimuli. Some of Allen and Kolers's subjects initially reported some multimodal motion effects, but they seemed to have perceived gamma motion (the light appeared to loom in and out from the location of the sound). Since the moving object in gamma motion was a light - not a multimodal object - it was not true intermodal multimodal apparent motion. Our subjects, on the other hand, were in a fully lit room, making gamma motion unlikely. Further, no sub-

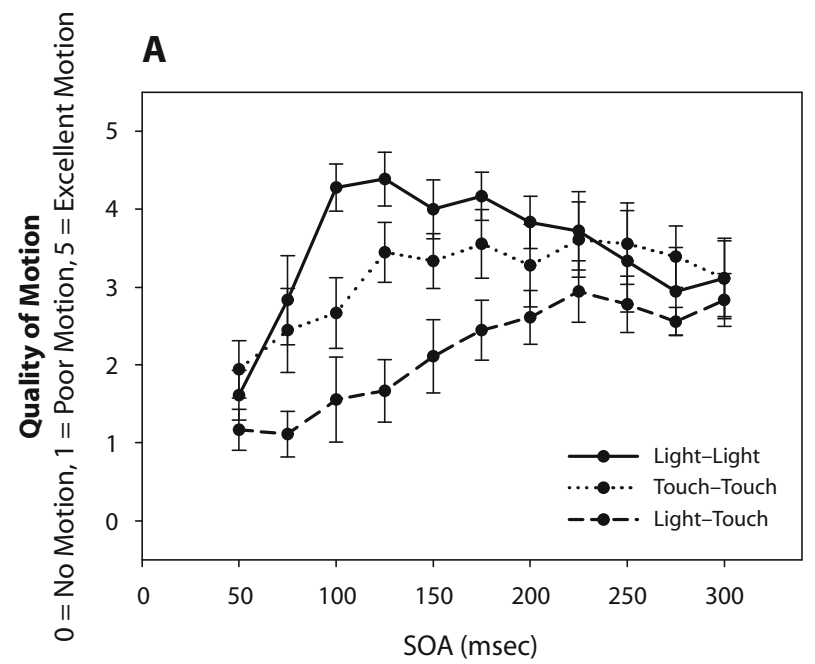

B Light-Touch

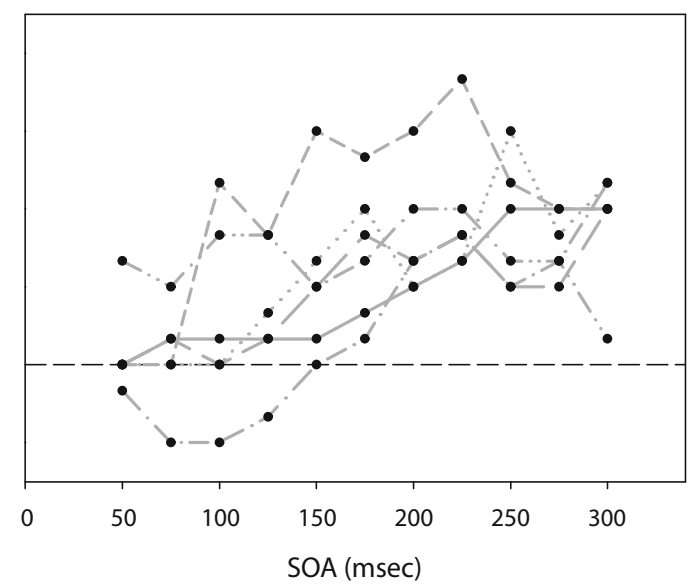

Figure 1. Quality of visual, tactile, and multimodal apparent motion. Subjects judged the quality of apparent motion on a 0-5 scale, where 0 indicated no motion perceived, 1 was poor, and 5 was excellent. (A) The quality of apparent motion between lights (solid line), between touches (dotted line), and between a light and a touch (dashed line), plotted as a function of stimulus onset asynchrony (SOA). (B) The quality judgment of visuotactile apparent motion for each subject as a function of SOA. At SOAs above 150 msec, all subjects perceived some multimodal apparent motion. 
jects reported the sensation of a single light moving to or from the location of the touch in the multimodal condition. Instead, subjects in the visuotactile condition reported perceiving some type of multimodal apparent motion, but they often described it as being "more causal" than the unimodal apparent motion. Our participants mainly interpreted their perception like a switch flicking on a light or like a cannon firing that was felt on one hand and then the flash from the landing explosive was seen on the other hand. This high-level interpretation may be evidence that multimodal motion occurs at a higher level of processing than unimodal apparent motion (Strybel \& Vatakis, 2004). On the other hand, "causal" multimodal apparent motion may, in fact, have a low-level basis, but subjects trying to express their perception may use high-level wording.

We have shown - for the first time - not just that multimodal visuotactile apparent motion exists, but also how the quality of its perception varies with SOA. In the next experiment, we tested whether the preferred delay for apparent motion varied with distance. Since, in Experi- ment 1 , tactile and multimodal apparent motion quality ratings were equal for a range of SOAs, in Experiment 2, we presented subjects with the same SOAs as in Experiment 1 , but used a forced-choice paradigm in order to obtain a "preferred delay." Doing so enabled us to directly test Korte's third law that the preferred delay would increase as a function of distance for visual, tactile, and multimodal apparent motion.

\section{EXPERIMENT 2 Preferred SOAs}

Experiment 1 established that multimodal apparent motion between visual and tactile stimuli exists and is comparable to unimodal apparent motion - at least at certain SOAs. In order to see whether multimodal apparent motion shared some of the properties of unimodal apparent motion, we varied the distance between the stimuli and noted the SOAs that gave the best sensation of motion. If multimodal apparent motion followed Korte's third law in
A Record the Stimulus Chosen as "Preferred"

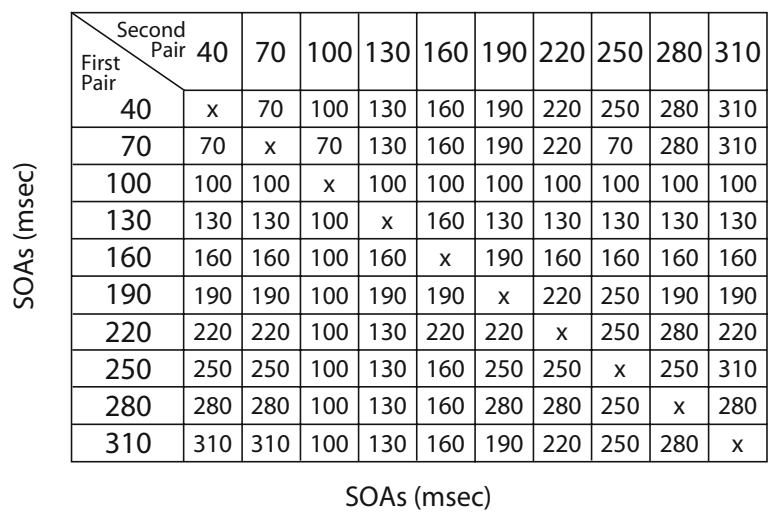

B

\begin{tabular}{|r|c|}
\hline SOA & $\begin{array}{c}\text { \# of Times } \\
\text { "Preferred" }\end{array}$ \\
\hline 40 & 0 \\
\hline 70 & 16 \\
\hline 100 & 27 \\
\hline 130 & 34 \\
\hline 160 & 30 \\
\hline 190 & 23 \\
\hline 220 & 20 \\
\hline 250 & 14 \\
\hline 280 & 10 \\
\hline 310 & 6 \\
\hline \multicolumn{2}{|c|}{ Max $=36$} \\
\hline
\end{tabular}

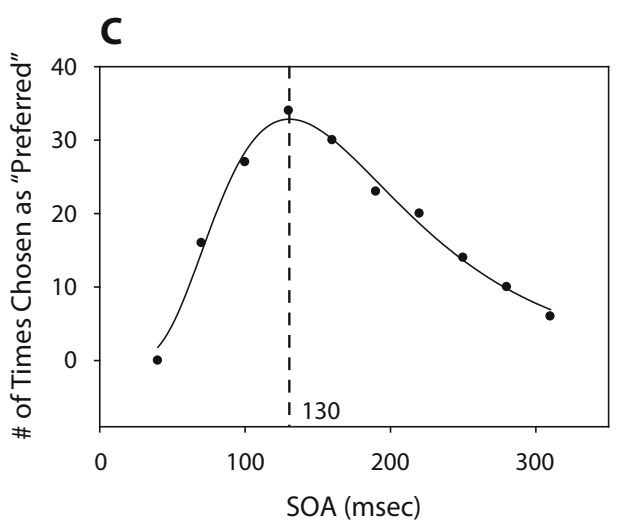

Figure 2. Preference method. (A) Each intersecting cell shows one example of which SOA was preferred by a subject out of the two possibilities. Each pair of delays was presented to the subject twice, so that each subject's data yielded two "A-charts." (B) The number of times each SOA was chosen was summed, up to a maximum of 36 . (C) The sum was then plotted as a function of SOA, and a logarithmic Gaussian fitted through the data. The peak of the Gaussian was taken as the preferred SOA, indicated by the vertical dashed line. 
the same way as unimodal apparent motion, it would suggest similarities in their mechanisms.

\section{Method}

Participants were physically positioned in the same way as they were in Experiment 1, maintaining fixation on a point in between two stimuli (one on either hand) at a viewing distance of $50 \mathrm{~cm}$. For each session, 10 SOAs were tested $(40,70,100,130,160,190,220$, $250,280,310 \mathrm{msec}$ ). Subjects were presented with two pairs of stimuli in succession and were instructed to indicate by means of foot pedals which of the pairs evoked the "most convincing" motion. The experiment was repeated with light-light, touch-touch, and lighttouch stimulus pairs that were 2,10 , and $56 \mathrm{~cm}$ apart $\left(2.3^{\circ}, 11.5^{\circ}\right.$, and $68.1^{\circ}$ ), resulting in a total of nine blocks, randomized across subjects in a counterbalanced design. Within a block, only one modality combination and one distance between the stimuli was used, so there were no comparisons made between different modalities or different distances. Each combination of delays was presented twice each way around (e.g., SOA of $40 \mathrm{msec}$ for the first pair followed by $100 \mathrm{msec}$ for the second pair, and $100 \mathrm{msec}$ followed by $40 \mathrm{msec}$ ). In each block, there were thus 180 comparisons (pairs with the same SOAs were not compared; see Figure 2A).

Each trial was initiated by pressing either of the foot pedals. The stimuli of the first pair then alternated back and forth with a particular SOA until the subject pressed a pedal. The second pair was then presented (with a different SOA), and it too repeated until the subject responded (subjects usually let the stimulus alternate one to four times). After the second pair was presented, the subject indicated which pair produced the more convincing percept of motion. The left foot pedal indicated that they preferred the first pair, and the right foot pedal indicated that they preferred the second.

Analysis. The pair that was chosen as "most convincing" was recorded (Figure 2A). The total number of times a particular SOA was chosen was scored. If a particular delay was selected as being more convincing than any of the others, then it would score a maximum of 36 (see Figure 2B). The number of times each SOA was chosen was then plotted as a function of the SOA (Figure 2C). A logarithmic Gaussian with the following function was then fitted:

Frequency $(\mathrm{SOA})=a * \exp \left\{-0.5 *\left[\ln \left(\mathrm{SOA} / \mathrm{SOA}_{0}\right) / b\right]^{2}\right\}$,

where $a$ is the maximum frequency, $\mathrm{SOA}_{0}$ is the preferred SOA, and $b$ is the standard deviation. A logarithmic function was chosen because of the skewed distribution inherent in this method. A preferred SOA value for each subject at each distance was thus found. Korte's third law predicts a linear increase in preferred SOA with distance. Therefore, we utilized linear contrast effects from the repeated measures ANOVA to detect the presence of a true positive relationship between distance and SOA (see Table 1 for the corresponding table of means).

\section{Results}

Light-light. Figure 3A plots the number of times each SOA was chosen as "preferred," averaged across the 6 subjects for visual apparent motion for lights separated by 2, 10 , or $56 \mathrm{~cm}$. The peak of the functions shown on the left indicates the mean preferred SOAs. The preferred SOAs for each subject at each distance are plotted on the right as a function of distance. Linear contrasts obtained from the ANOVA confirmed that the preferred SOA increased with distance $\left[F_{\text {linear }}(1,5)=17.90, p=.004 ; \eta_{\mathrm{p}}^{2}=.782\right]$. Nearly $80 \%$ of the variability in the preferred SOA can be accounted for by the distance between the two stimuli-a large effect of distance.

Touch-touch. Figure 3B plots the number of times each SOA was chosen as "preferred," averaged across the 6 subjects for tactile apparent motion between touches on the hands separated by 2,10 , or $56 \mathrm{~cm}$. For unimodal apparent motion between two touches, there was no significant effect of interstimulus distance on the preferred SOA when we looked over the full range of distances tested $(2-56 \mathrm{~cm})\left[F_{\text {linear }}(1,5)=1.09, p=.17 ; \eta_{\mathrm{p}}^{2}=.18\right]$. However, following observation of the data, the same test was conducted on the data collected at just the two shorter distances $(2$ and $10 \mathrm{~cm}$ ). A significant and moderately sized linear effect was indeed present over this smaller range of distances $\left[F_{\text {linear }}(1,5)=4.58, p=.042 ; \eta_{\mathrm{p}}^{2}=.48\right]$. At smaller distances, nearly $50 \%$ of the variability in preferred SOA can be explained by distance.

Light-touch. Figure 3C plots the number of times each SOA was chosen as "preferred," averaged across the 6 subjects for multimodal apparent motion between a light on one hand and a touch on the other, separated by 2 , 10 , or $56 \mathrm{~cm}$. For multimodal apparent motion between a touch and a light, an ANOVA confirmed that the preferred SOA did not change with distance $\left[F_{\text {linear }}(1,5)=0.01, p=\right.$ $\left..46 ; \eta_{\mathrm{p}}^{2}=.003\right]$.

\section{Discussion}

Light-light. Our suprathreshold measure of apparent motion confirmed that the preferred SOA increased with distance. This variation with distance was first suggested by Korte in 1915 and has been confirmed many times since (see, e.g., Koffka, 1931; Kolers, 1972). Since angular and linear distance have always covaried in these studies, we cannot tell which factor affected the preferred delay for apparent motion.

Touch-touch. Tactile apparent motion has previously been investigated for touches along the same body part (Burtt, 1917b; Kirman, 1974b; Lakatos \& Shepard, 1997) and across empty space between the two hands or thighs (Sherrick, 1968; Sherrick \& Rogers, 1966). Distance was shown to affect preferred SOA for tactile apparent motion along the same body part, but was not systematically

Table 1

Means (in Milliseconds) and Corresponding Standard Errors for Preferred SOAs (for Apparent Motion Evoked at Three Distances) and for Threshold SOAs (for Detecting the Direction of Motion at Three Distances)

\begin{tabular}{|c|c|c|c|c|c|c|c|c|c|c|c|c|}
\hline \multirow[b]{3}{*}{ Motion Type } & \multicolumn{6}{|c|}{ Preferred SOA } & \multicolumn{6}{|c|}{ Threshold SOA } \\
\hline & \multicolumn{2}{|c|}{$2 \mathrm{~cm}$} & \multicolumn{2}{|c|}{$10 \mathrm{~cm}$} & \multicolumn{2}{|c|}{$56 \mathrm{~cm}$} & \multicolumn{2}{|c|}{$2 \mathrm{~cm}$} & \multicolumn{2}{|c|}{$6 \mathrm{~cm}$} & \multicolumn{2}{|c|}{$18 \mathrm{~cm}$} \\
\hline & $M$ & $S E$ & $M$ & $S E$ & $M$ & $S E$ & $M$ & $S E$ & $M$ & $S E$ & $M$ & $S E$ \\
\hline Visual & 137.5 & 15.7 & 165.9 & 25.3 & 248.1 & 70.8 & 9.3 & 2.3 & 11.9 & 4.1 & 28.1 & 6.3 \\
\hline Tactile & 184.4 & 49.0 & 230.8 & 88.6 & 170.9 & 35.0 & 34.0 & 4.3 & 39.1 & 8.0 & 47.2 & 7.1 \\
\hline Multimodal & 192.9 & 109.3 & 181.6 & $\begin{array}{l}56.0 \\
56.8\end{array}$ & 188.4 & 65.2 & 56.6 & 7.3 & 50.4 & 6.8 & 52.4 & 5.9 \\
\hline
\end{tabular}


A

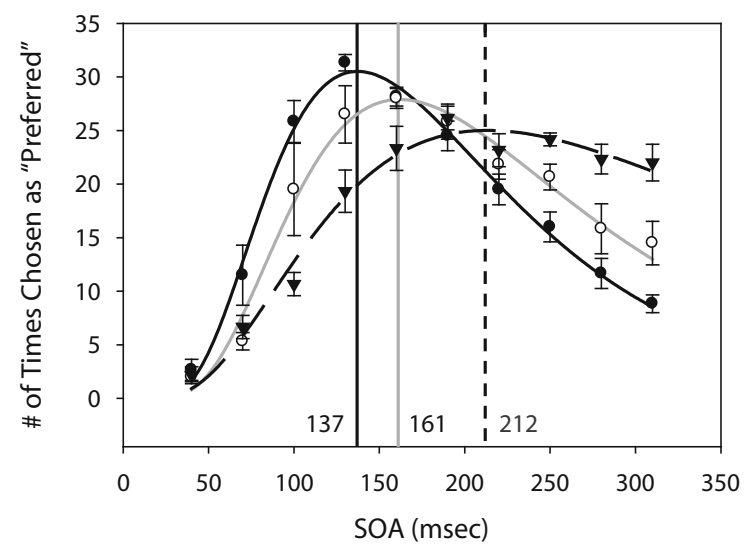

Light-Light

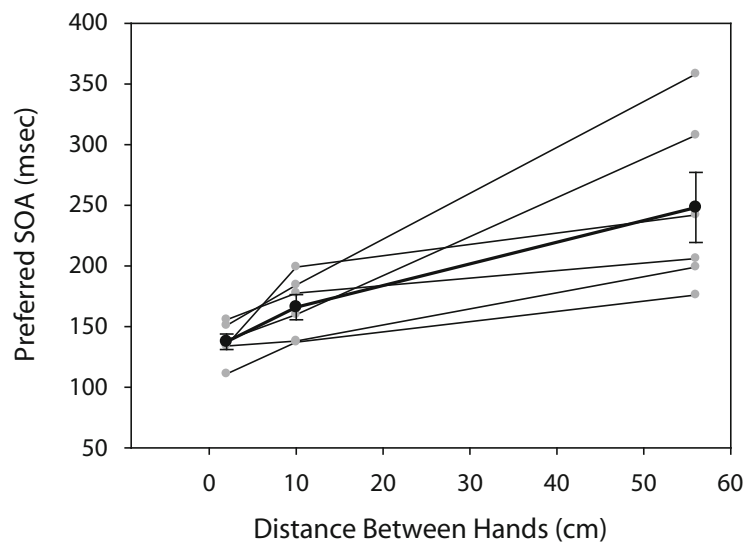

B

Touch-Touch
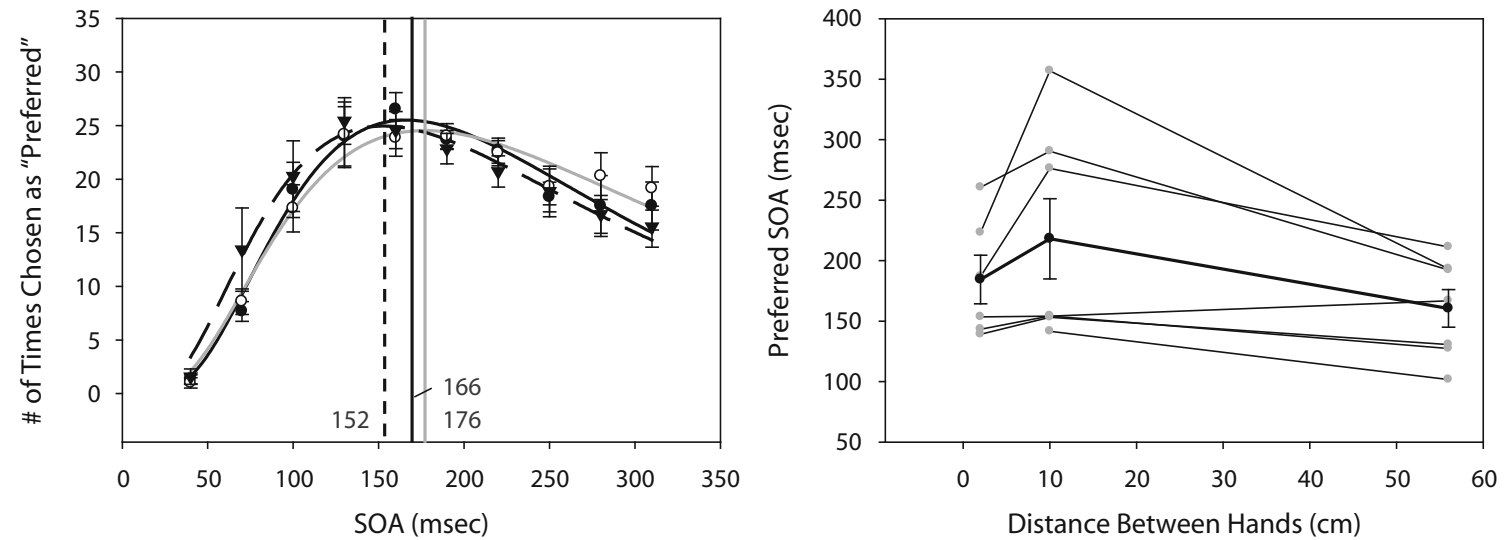

C

Light-Touch
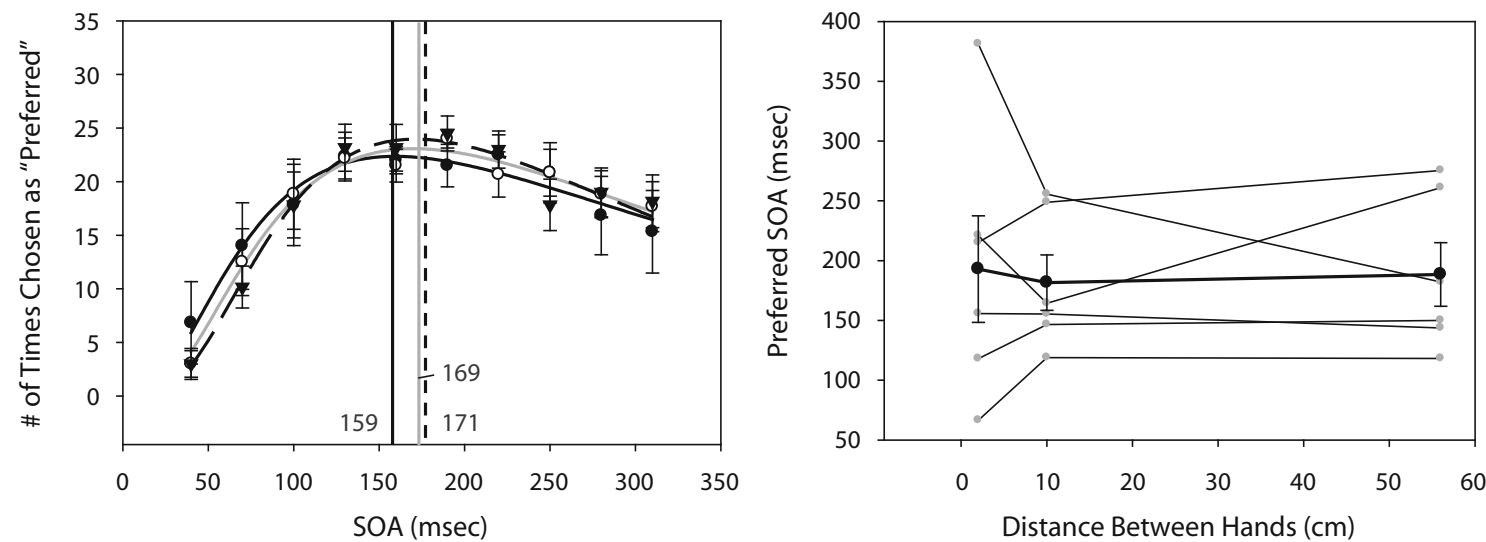

Figure 3. SOAs for preferred apparent motion. The graphs on the left plot the average number of times that each SOA was chosen as "preferred," as a function of the SOA, with standard error bars for each apparent motion type (light-light; touch-touch; light-touch). Best-fit logarithmic Gaussians are plotted through the data for each distance (filled circles, solid black curve, 2 cm; open circles, gray curve, $10 \mathrm{~cm}$; filled triangles, dashed curve, $56 \mathrm{~cm}$ ), and the peak of each Gaussian is indicated by a matching vertical line. The thinline plots on the right are the preferred SOAs (peaks of the Gaussians) for each subject plotted as a function of the distance between the stimuli. The thick lines are plotted through the average preferred SOAs with standard error bars. 
investigated across empty space. The present experiment is the first to show that the perception of tactile apparent motion varies with distance across the empty space between the hands - at least up to a separation of $10 \mathrm{~cm}$. This requires that subjects integrate knowledge of where their hands are (proprioceptive cues) with the perception of motion. The perception of motion over a much larger distance $(56 \mathrm{~cm})$ may not follow Korte's third law, because it may be generated by a different mechanism than apparent motion over smaller distances. The moderate effect size for touch-touch over smaller distances $\left(\eta_{\mathrm{p}}^{2}=.48\right)$ is smaller than the large effect size for light-light $\left(\eta_{\mathrm{p}}^{2}=.78\right)$, which suggests that more factors affect the quality of motion for touch-touch than for light-light. If the light-light and touch-touch apparent motions have different factors affecting them, or different weightings for each factor, then it suggests that they have different mechanisms.

Light-touch. A second new finding that emerged from Experiment 2 is that the preferred SOA for multimodal apparent motion between a touch and a light was not affected by the distance that separated the stimuli, even over a range that affected both unimodal visual and tactile apparent motion. Since Korte's third law was thus not supported by the data from the visuotactile condition, it seems that the mechanism responsible for the perception of multimodal motion is different from the unimodal mechanisms. The mechanism responsible for the perception of visuotactile apparent motion may share similarities with long-distance tactile apparent motion and auditory apparent motion mechanisms, which also do not follow Korte's third law.

\section{Comparison of Unimodal and Multimodal Apparent Motion}

Although visual apparent motion and tactile apparent motion (over modest distances) were both affected by the distance between the stimuli, the size of the effect was different. The two modalities did not have the same preferred SOA, and the slopes of the increases of SOA with distance were also not the same. However, the visual and tactile mechanisms do share some characteristics. For example, they are both described by Korte's third law, whereas the multimodal and auditory mechanisms are not.

In order to further explore the similarities and differences between unimodal and multimodal motion processing, Experiment 3 looked at the characteristics of motion perception at threshold.

\section{EXPERIMENT 3 Threshold SOAs}

Korte's (1915) laws were originally developed to describe suprathreshold preferred apparent motion. However, Lakatos and Shepard (1997) showed that the threshold for detecting the direction of apparent motion also varies with distance, in accordance with Korte's third law. Unlike preferred motion, thresholds seemed to follow the rule for a broader range of modalities including visual, tactile, and auditory apparent motion. This result suggests that-at least for auditory apparent motion - there may be separate mechanisms that process suprathreshold and threshold motion. The results of Experiment 2 suggested that the characteristics of the for-

A
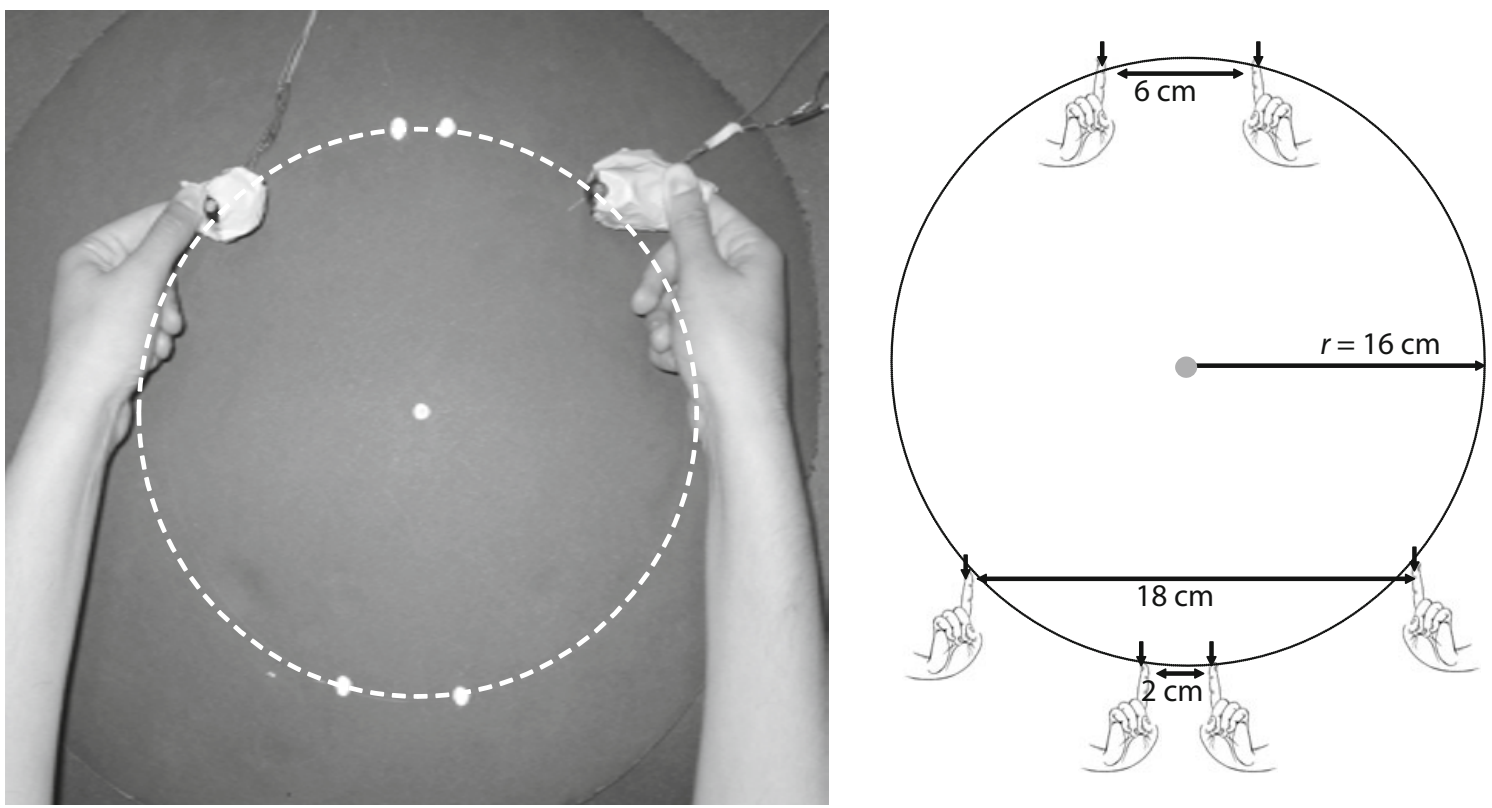

Figure 4. Setup for Experiment 3. (A) Photograph of a subject's hands placed at points on a circle. The dashed line was not visible to the subject and is added to indicate the positions at which they held their hands. (B) All points on the circle had an eccentricity of $18^{\circ}$ from the central fixation point. Stimuli were tested at separations of 2,6 , and $18 \mathrm{~cm}\left(2.3^{\circ}, 6.9^{\circ}\right.$, and $20.4^{\circ}$, respectively). 
mer mechanism are not universal; however, the characteristics for the latter may be. We therefore measured direction thresholds for stimuli at various separations to see whether visual, tactile, and visuotactile apparent motion SOA thresholds varied linearly with distance.

\section{Method}

The threshold SOAs for direction discrimination of apparent motion between visual pairs, tactile pairs, and crossmodal visuotactile pairs were measured for stimulus separations of 2, 6, and $18 \mathrm{~cm}$ (corresponding to visual angles of $2.3^{\circ}, 6.9^{\circ}$, and $20.7^{\circ}$ at the viewing distance of $50 \mathrm{~cm}$ ). Subjects fixated the center of a $16-\mathrm{cm}$ radius circle with their hands (with stimuli attached; see the General Method section) arranged at left-right symmetrical points around the circle (see Figure 4). In this way, an eccentricity of $18^{\circ}$ was maintained while the interstimulus distances and corresponding visual angles were varied.

Three combinations of stimuli were tested: light left-light right, touch left-touch right, and light left-touch right, all randomly interleaved. There were eight repetitions of $23 \operatorname{SOAs}( \pm 110, \pm 100, \pm 90$, $\pm 80, \pm 70, \pm 60, \pm 50, \pm 40, \pm 30, \pm 20, \pm 10$, and $0 \mathrm{msec}$ ), where a negative delay indicates that the left stimulus was presented first. Each stimulus pair was presented once, and the subject indicated the perceived direction of motion: The stimuli were not repeated as they were in Experiments 1 and 2, since the task was to discriminate direction which would not be possible if the stimulus was bouncing back and forth. Participants reported the perceived direction of motion (left or right) by use of the left and right foot pedals. The threshold was defined as the point at which the direction was correctly identified $75 \%$ of the time.

Analysis. The percentage of time that subjects responded "left" was plotted as a function of the SOA (Figure 5A). The data were fitted by a cumulative Gaussian:

percentage of times

reported "left" $(\mathrm{SOA})=100 /\{1+\exp [-(\mathrm{SOA}-\mathrm{PSE}) / b]\}$,

where $b$ is the standard deviation and the point of subjective equality (PSE) is the SOA at which subjects were equally likely to perceive motion going left or right (the point of subjective equality). The PSE was not necessarily at 0 , especially for multimodal motion, because of the different processing times for visual and tactile stimuli (Har- rar \& Harris, 2005). The PSE of each subject was therefore used as the zero point at which no motion was perceived, and the other points were expressed as the absolute time difference to the PSE. Thus, an SOA of $-100 \mathrm{msec}$ with a PSE of $-11 \mathrm{msec}$ would be scored as a relevant time difference of $89 \mathrm{msec}$. Responses could thus be converted to percent correct (Figure 5B) and plotted as a function of the relevant time difference. A second cumulative Gaussian - forced between $50 \%$ and $100 \%$ - was fitted through these data from which the $75 \%$-correct point was determined (see Lakatos \& Shepard, 1997). The resulting direction thresholds were plotted as a function of the distance between the stimuli. The linear contrasts derived from the repeated measures ANOVA determined whether there was a significant linear effect of distance on threshold (see Table 1 for the corresponding table of means). One subject's data were outliers $(z$ score $>3)$ and therefore were removed; the removal of this subject's data did not affect the significance of any of the findings reported.

\section{Results and Discussion}

Light-light. Figure 6A shows the mean psychometric functions on the left and the threshold values plotted as a function of distance for each subject and for the mean on the right. For visual apparent motion, the threshold for direction discrimination increased as the distance between the two lights increased $\left[F_{\text {linear }}(1,5)=10.65, p=.011\right]$, with a moderate effect size $\left(\eta_{\mathrm{p}}^{2}=.68\right)$ in accordance with Korte's third law. This pattern was similar to that found for suprathreshold visual apparent motion in Experiment 2; however, the threshold SOAs were much shorter than the "preferred" SOAs. This experiment specifically controlled for the possible confound of eccentricity, which did not have an effect on the linear relationship between distance and delay.

Touch-touch. Figure 6B shows the mean psychometric functions on the left and the threshold values as a function of distance for each subject and for the means on the right. For tactile apparent motion, as the distance between the two hands was increased, the threshold SOA also increased
A

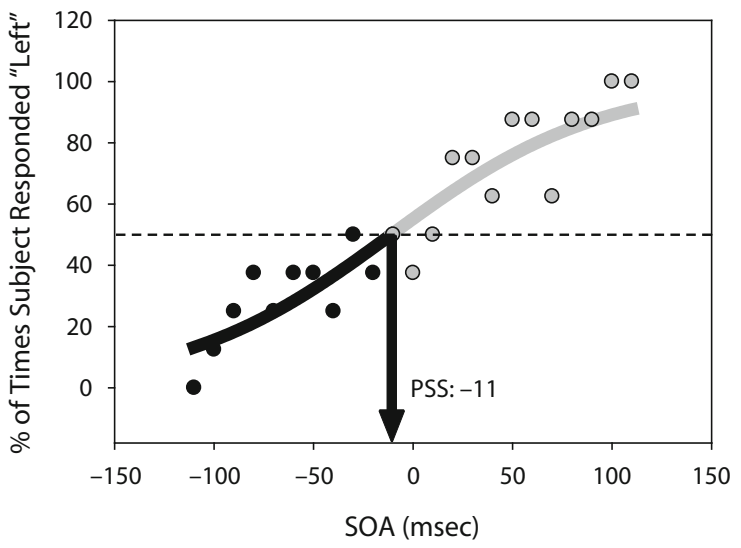

B

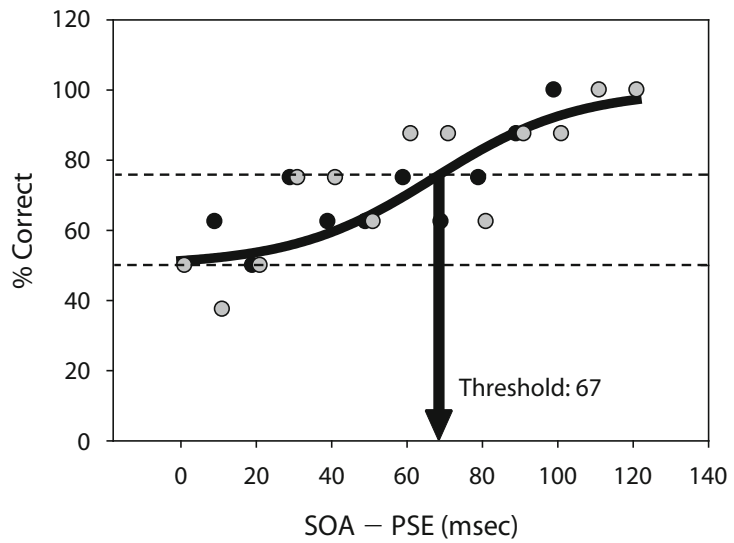

Figure 5. Analysis for Experiment 3. (A) A cumulative Gaussian function was fitted to the number of times that subjects responded "left" as a function of the SOA. The point on the curve where subjects were equally likely to say "left" or "right" (the 50\% point) represented the individual subject's point of subjective equality (PSE). (B) For each subject, the percent correct (gray circles, correct response is "left"; black circles, correct response is "right") was then plotted as a function of the absolute difference between the SOA and PSE. Another cumulative Gaussian was fitted to the percent correct responses, forced between $50 \%$ and $100 \%$. Threshold was defined as the SOA required for the subjects to correctly identify the direction of apparent motion $75 \%$ of the time. 
A

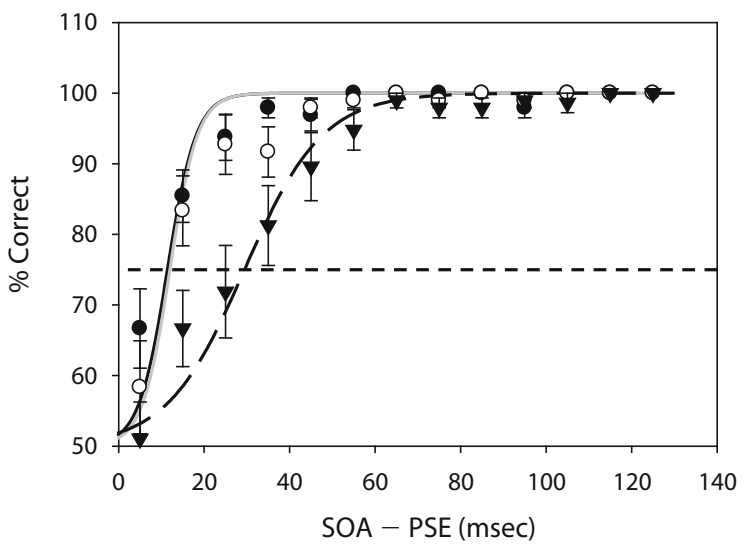

Light-Light

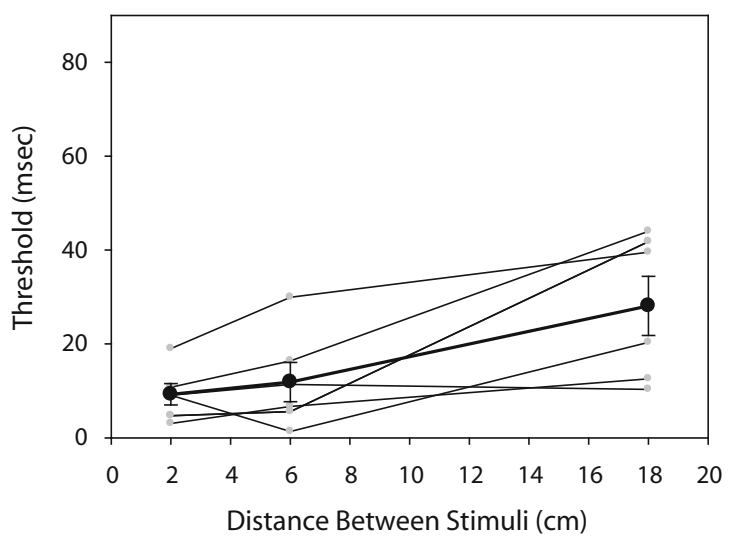

B

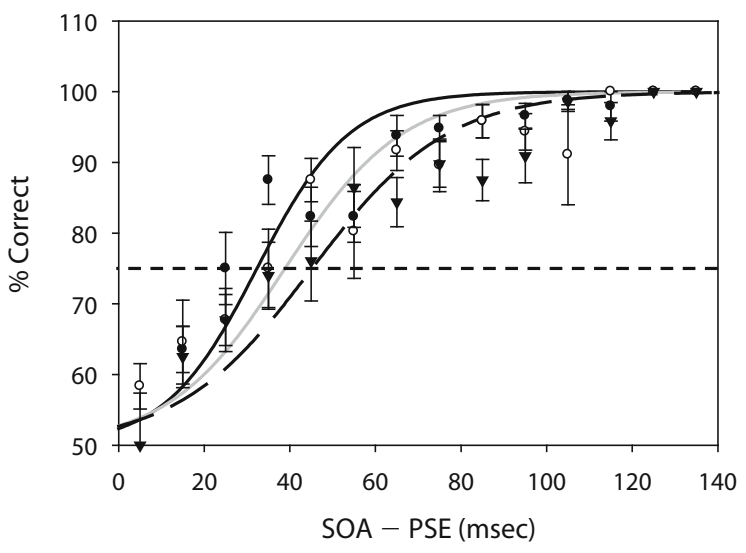

Touch-Touch

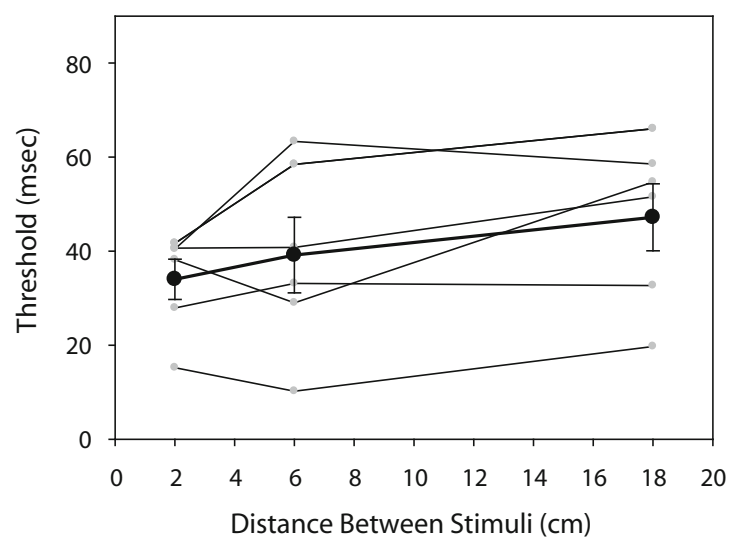

C

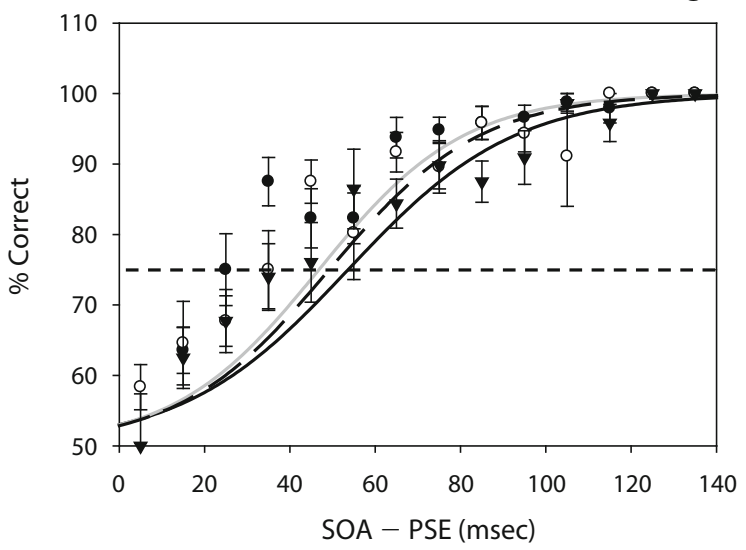

Light-Touch

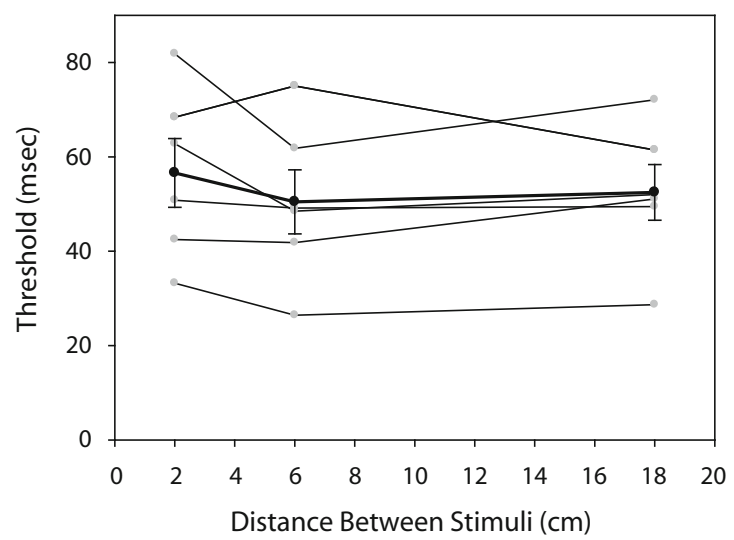

Figure 6. Thresholds of apparent motion vary with distance. Left panels: The percentage correct is plotted as a function of the relevant timing difference (the absolute difference between the SOA and the PSE as derived in Figure 5 and described in the text). Cumulative Gaussians are plotted through the data for each distance (shown as averages with standard error bars: filled circles and black lines, $2 \mathrm{~cm}$; open circles and gray lines, $6 \mathrm{~cm}$; filled triangles and dashed lines, $18 \mathrm{~cm}$ ). The $75 \%$ points (indicated by the horizontal dashed lines) were taken as the thresholds. Right panels: Thresholds are plotted as a function of distance for each subject (thin lines), and for all the data pooled (thick lines), with standard error bars. 
$\left[F_{\text {linear }}(1,5)=16.72, p=.005\right]$, with a moderately large effect size $\left(\eta_{\mathrm{p}}^{2}=.77\right.$ - even larger than that for light-light).

The linear relationship (between the threshold for determining the direction of tactile apparent motion and distance) applied to the whole range of distances tested in Experiment $3(18 \mathrm{~cm})$. Similar findings were reported by Lakatos and Shepard (1997), who investigated tactile apparent motion with stimulators separated over the palm of the hand or around the head. In their experiments, the distances between the stimulators were determined exclusively by where they were on the body. In our experiments, the distance between the two stimulators was varied by moving the hands apart; the distance between the stimulators in our experiments was thus determined by both the location of the hands in space (proprioception) and the body location stimulated. As in Experiment 2, we found that for tactile apparent motion, proprioceptive knowledge is integrated with somatosensory information to produce the percept of movement between the two hands (see also Dassonville, 1995; Sanabria, SotoFaraco, \& Spence, 2005).

For direction thresholds, the effect sizes were similar for light-light (.68) and for touch-touch (.77), suggesting that around $70 \%$ of the variability in thresholds can be explained by the distance between the stimuli. Here, touch-touch does not seem to have more factors affecting the threshold than light-light, as it did in preferred SOA judgments.

Light-touch. For visuotactile apparent motion, the threshold SOA did not significantly change as the distance between the two hands changed $\left[F_{\text {linear }}(1,5)=2.03, p=\right.$ $.102 ; \eta_{\mathrm{p}}^{2}=.29$; see Figure 6C].

Visuotactile apparent motion had a threshold delay for direction discrimination of around $50 \mathrm{msec}$ that did not vary. ${ }^{1}$ This lack of an effect of distance was similar to the results of Experiment 2 concerning suprathreshold multimodal apparent motion although, of course, the threshold SOAs (about $50 \mathrm{msec}$ ) were much smaller than the "preferred" values (about $175 \mathrm{msec}$ ).

\section{GENERAL DISCUSSION}

These experiments have confirmed that multimodal motion between lights and touches can occur. Although apparent motion between two lights obeys Korte's third law, in the touch system this is only true for separations up to around $20 \mathrm{~cm}$ (when stimuli are on different hands). Multimodal apparent motion between a touch and a light does not seem to depend on distance.

The SOA for correctly determining the direction of visual or tactile apparent motion increases with an increase in distance and is unaffected for visuotactile motion by distance. In contrast, the threshold SOA for correctly determining temporal order of tactile (Shore, Gray, Spry, \& Spence, 2005) or visuotactile (Spence, Baddeley, Zampini, James, \& Shore, 2003; Spence, Shore, \& Klein, 2001) stimuli decreases with an increase in distance. This result suggests that subjects are not simply responding to the temporal order of the stimuli. Other studies testing the robustness of the multimodal visuotactile apparent motion can be done, such as habituation and priming.

\section{Angular Versus Linear}

The methodology used in Experiment 3 rules out effects of eccentricity, but angular versus linear distance are still confounded. Koffka (1931) and Korte (1915) also confounded the angular and the physical distance between their stimuli. Further experiments need to be done to examine whether the effect depends on either or both of these variables. Such an experiment would need to pay careful attention to controlling for variations in intensity, stimulus size, and distance cues (binocular and monocular). If receptive fields in touch are analogous to angles in vision, then similar experiments could be done for tactile apparent motion on different body parts with varying receptive field sizes.

\section{A Suprasensory Motion Mechanism?}

The idea of a supramodal, motion-extracting system was postulated by Aristotle (trans. 1984) in his notion of a sensus communis, a "common sensible of movement ... that [is] not special to any one sense, but [is] common to all."' Just as visual and vestibular cues are integrated by a common intramodal self-motion detecting mechanism to evoke a sense of vection, might there also be integration of visual and tactile motion signals by a motion-sensitive sensus communis? Within vision, apparent motion can be experienced between objects with clearly differing forms (Burt \& Sperling, 1981); it is not necessary that the stimuli match. From observations of this kind, Kolers (1972) concluded that "the visual system responds to locations of stimulation and infers or creates changes of figure to resolve that disparity" (p. 57). If similarity of form is not necessary for visual apparent motion, then it seems reasonable to suggest that multimodal stimuli could converge into a single perception of motion.

Multimodal apparent motion might be created by a process of multisensory integration (see Spence, Sanabria, \& Soto-Faraco, 2007, for a historical discussion of multimodal apparent motion); we suggest two such models of integration. First, multisensory integration can occur in space (Craig, 2005) and/or in time (spatial ventriloquism, Thurlow \& Jack, 1973; temporal ventriloquism, Aschersleben \& Bertelson, 2003). The result of such integrations could then feed into a single supramodal motion mechanism. In such a mechanism, the location and temporal cues are extracted from the sensory input and are not tagged as originating from any particular modality. However, the differences between unimodal and multimodal apparent motion described in our experiments suggest that it is unlikely that vision and touch share a single motion detecting mechanism.

Alternatively, suprasensory multimodal motion could result from a combination of unimodal motion (Lyons, Sanabria, Vatakis, \& Spence, 2006). Such a model does not require that visual, tactile, and multimodal apparent motion follow the same laws, since each has a distinct motion mechanism. The multimodal combination could be either statistically optimal (Soto-Faraco, Spence, \& Kingstone, 2004) or such that visual motion dominates tactile motion perception (Craig, 2006). Our present data indeed suggest separate motion mechanisms for multimodal and unimodal 
motion processing. Explaining the mechanism for motion processing within and across the different senses will hold the key to understanding how redundant information about space and time are integrated across the senses.

\section{AUTHOR NOTE}

We gratefully acknowledge the support of the Natural Sciences and Engineering Research Council (NSERC) of Canada to L.R.H. V.H. and R.W. also hold NSERC scholarships. Address correspondence to V. Harrar, Department of Psychology, York University, 4700 Keele St., Toronto, ON, M3J 1P3 Canada (e-mail: vharrar@yorku.ca).

\section{REFERENCE}

Allen, P. G., \& Kolers, P. A. (1981). Sensory specificity of apparent motion. Journal of Experimental Psychology: Human Perception \& Performance, 7, 1318-1328.

Aristotle (1984). De anima [On the soul]. In J. Barnes (Ed.), The complete works of Aristotle: The revised Oxford translation (Vol. 1, pp. 641-692). Princeton, NJ: Princeton University Press. (Original work published c. $350 \mathrm{BCE}$ )

Aschersleben, G., \& Bertelson, P. (2003). Temporal ventriloquism: Crossmodal interaction on the time dimension. 2: Evidence from sensorimotor synchronization. International Journal of Psychophysiology, 50, 157-163.

BurT, P., \& Sperling, G. (1981). Time, distance, and feature trade-offs in visual apparent motion. Psychological Review, 88, 171-195.

BurTt, H. (1917a). Auditory illusions of movement: A preliminary study. Journal of Experimental Psychology, 2, 63-74.

BurTt, H. (1917b). Tactile illusions of movement. Journal of Experimental Psychology, 2, 371-385.

Craig, J. C. (2005). The trajectory effect in intermodal temporal order judgments. Perception, 34, 357-370.

Craig, J. C. (2006). Visual motion interferes with tactile motion perception. Perception, 35, 351-367.

DassonVILLE, P. (1995). Haptic localization and the internal representation of the hand in space. Experimental Brain Research, 106, 434-448.

Duhamel, J. R., Colby, C. L., \& Goldberg, M. E. (1998). Ventral intraparietal area of the macaque: Congruent visual and somatic response properties. Journal of Neurophysiology, 79, 126-136.

Harrar, V., \& Harris, L. R. (2005). Simultaneity constancy: Detecting events with touch and vision. Experimental Brain Research, 166, 465-473.

KIRMan, J. H. (1974a). Tactile apparent movement: The effects of interstimulus onset interval and stimulus duration. Perception \& Psychophysics, 15, 1-6.

Kirman, J. H. (1974b). Tactile apparent movement: The effects of number of stimulators. Journal of Experimental Psychology, 103, $1175-1180$

KoffKA, K. (1931). Die Wahrnehmung von Bewegung. In A. Bethe (Ed.), Handbuch der normalen und pathologische Physiologie: Vol. 12, part 2 (pp. 1166-1214). Berlin: Springer.

Kolers, P. A. (1972). Aspects of motion perception. Oxford: Pergamon Press.

KoRTE, A. (1915). Kinematoskopische Untersuchungen. Zeitschrift für Psychologie, 72, 194-296.

LAKatos, S., \& Shepard, R. N. (1997). Constraints common to apparent motion in visual, tactile, and auditory space. Journal of Experimental Psychology: Human Perception \& Performance, 23, 1050-1060.
Lyons, G., Sanabria, D., Vatakis, A., \& Spence, C. (2006). The modulation of crossmodal integration by unimodal perceptual grouping: A visuotactile apparent motion study. Experimental Brain Research, 174, 510-516.

Marks, L. E. (1978). The unity of the senses. New York: Academic Press.

Sanabria, D., Soto-Faraco, S., \& Spence, C. (2005). Spatiotemporal interactions between audition and touch depend on hand posture. $E x$ perimental Brain Research, 165, 505-514.

SHERrick, C. E. (1968). Bilateral apparent haptic movement. Perception \& Psychophysics, 4, 159-162.

Sherrick, C. E., \& Rogers, R. (1966). Apparent haptic movement. Perception \& Psychophysics, 1, 175-180.

Shore, D. I., Gray, K., Spry, E., \& Spence, C. (2005). Spatial modulation of tactile temporal-order judgments. Perception, 34, 1251-1262.

Soto-Faraco, S., Spence, C., \& Kingstone, A. (2004). Congruency effects between auditory and tactile motion: Extending the phenomenon of cross-modal dynamic capture. Cognitive, Affective, \& Behavioral Neuroscience, 4, 208-217.

Spence, C., Baddeley, R., Zampini, M., James, R., \& Shore, D. I. (2003). Multisensory temporal order judgments: When two locations are better than one. Perception \& Psychophysics, 65, 318-328.

Spence, C., Sanabria, D., \& Soto-Faraco, S. (2007). Intersensory Gestalten and crossmodal scene perception. In K. Noguchi (Ed.), Psychology of beauty and Kansei: New horizons of Gestalt perception (pp. 519-579). Tokyo: Fuzanbo International.

Spence, C., Shore, D. I., \& Klein, R. M. (2001). Multisensory prior entry. Journal of Experimental Psychology: General, 130, 799-832.

Strybel, T. Z., Manligas, C. L., Chan, O., \& Perrott, D. R. (1990). A comparison of the effects of spatial separation on apparent motion in the auditory and visual modalities. Perception \& Psychophysics, $47,439-448$

Strybel, T. Z., \& Vatakis, A. (2004). A comparison of auditory and visual apparent motion presented individually and with crossmodal moving distractors. Perception, 33, 1033-1048.

Thurlow, W. R., \& JACK, C. E. (1973). Certain determinants of the "ventriloquism effect." Perceptual \& Motor Skills, 36, 1171-1184.

Zapparoli, G. C., \& Reatto, L. L. (1969). The apparent movement between visual and acoustic stimulus and the problem of intermodal relations. Acta Psychologica, 29, 256-267.

\section{NOTES}

1. Although no significant effect of distance was found, a moderate effect size was detected, suggesting that our sample size might not have been large enough to detect the presence of an effect of distance. Therefore, we decided to further explore the possibility of an effect in this condition. The same experiment had been conducted on 4 left- and 7 right-handed subjects, and no difference between the two groups had been found (right-handed subjects reported above, one outlier removed). We now pooled the data from the two groups, for a total of 10 subjects. With the larger number of subjects, the possible effect disappeared completely $\left[F_{\text {linear }}(1,9)=0.001, p=.49 ; \eta_{\mathrm{p}}^{2}=.00\right]$. We therefore conclude that, overall, there was no effect of distance on visuotactile apparent motion threshold.

2. Aristotle (trans. 1984), 418a (p. 665). See Marks (1978) for a history of the sensus communis concept.

(Manuscript received August 7, 2006; revision accepted for publication January 21, 2008.) 